\title{
Chemoprävention beim Bronchialkarzinom: Überblick über bisherige Studien, Ergebnisse und zukünftige Entwicklungen
}

M. Heckmayr

\author{
Chemoprevention of Lung Cancer. A Review about Previous Studies, Results \\ and Future Developments
}

\section{Zusammenfassung}

Trotz verbesserter Früherkennungsmaßnahmen beim Bronchialkarzinom steigen die Mortalitätsraten weltweit und die 5-Jahres-Überlebensrate liegt weiterhin unter $10 \%$. Mit der Chemoprävention besteht die Möglichkeit, mit Hilfe natürlicher oder synthetischer Substanzen in den Prozess der Kanzerogenese so einzugreifen, dass entweder die Schädigung der DNA verhindert oder aber die Proliferation bereits vorhandener prämaligner Zellen unterbunden wird. Chemoprävention beim Bronchialkarzinom bedeutet zwar primär Aufgabe des Nikotinkonsums, viele Ex-Raucher sind aber über einen längeren Zeitraum weiterhin einem erhöhten Karzinomrisiko ausgesetzt (sekundäre Prävention). In den letzten 20 Jahren sind detaillierte Kenntnisse über den Mechanismus der Karzinogenese bekannt geworden, die den gezielten Einsatz von Chemoprotektiva erleichtern. Neben den klassischen Chemoprotektiva, zu denen Vitamin A, $\beta$-Carotin, die Vitamine E, C und B12 ebenso wie Selen gehören, wurden in den letzten Jahren neuere Substanzen entwickelt, wie die Retinamide als synthetische Verbindungen des Vitamin A, Dithiole, Cyclooxygenase-Inhibitoren, epidermale Wachstumsfaktoren u. a., deren Mechanismen interessante Ansatzpunkte der Chemoprävention liefern. Die Entwicklung von Biomarkern (Genom-, Proliferations- und Differenzierungsmarker), mit deren Hilfe verschiedenen Phasen im Prozess der Krebsentstehung verfolgt werden können, erlaubt es, Studien zur Prävention schneller und effektiver durchzuführen. Nach Optimierung entsprechender Studiendesigns ist zu hoffen, dass in absehbarer Zeit klini-

\section{Abstract}

Despite improvements in the early detection of lung tumors, mortality from this type of disease is increasing world wide and the 5-year-survival rate still ranges below $10 \%$. The approach of chemoprevention offers the possibility to interfere with the process of cancerogenesis by the use of natural or synthetic chemical compounds and either to prevent DNA damage or to stepp the proliferation of premalignant cells that are already in place. With regard to bronchial carcinoma, chemoprevention in the first place implies cessation of smoking but the currently used procedures are not extremely efficient and many ex-smokers experience an increased risk of acquiring a lung tumor over a prolonged period of time (secondary prevention). In the past 20 years many details of the process of tumorigenesis have been revealed and this knowledge has promoted the targeted use of chemopretective compounds. In addition to the classics in the fields, such as vitamin A, $\beta$-carotene, vitamins E, C and B12 as well as selenium, the last years have brought the development of new compounds, such as retinoids, dithiols, cyclooxygenase inhibitors, epidermal growth factors and others, the mechanismen of action of which provide interesting new approaches. In addition, the introduction of biomarkers, either genetic alterations or proliferation or differentiation, allows to monitor the process of tumorigenesis in its different stages, from early to late, and thereby offers the perspective to perform studies on chemoprevention more rapidly and effectively as in previous years. Thus, in combination with optimized, controlled study designs it is to be expected that in

Institutsangaben

Zentrum für Pneumologie und Thoraxchirurgie Krankenhaus Großhansdorf

Anmerkung

Herrn Professor Dr. H. Magnussen zum 60. Geburtstag gewidmet.

Korrespondenzadiresse

Dr. med. Marlene Heckmayr · Krankenhaus Großhansdorf, Zentrum für Pneumologie und Thoraxchirurgie · Abteilung für Pneumologische Onkologie · Leiter Dr. med. U. Gatzemeier · Wöhrendamm 80 .

22927 Großhansdorf ·E-mail: m.heckmayr@gmx.net

Eingang: 10. April 2004 · Angenommen: 6. Mai 2004

Bibliografie

Pneumologie 2004; 58: 408-415 • @ Georg Thieme Verlag KG Stuttgart · New York

DOI 10.1055/s-2004-818501

ISSN 0934-8387 
sche Studien mit Chemoprotektiva effizient bei chronischen Rauchern und Ex-Rauchern durchgeführt werden können. near future clinical studies on the effects on chemoprotective compounds will yield decisive data on their efficacy in chronic smokers as well as ex-smokers.

\section{Einleitung}

Chemoprävention bei Tumoren bedeutet einen Eingriff in den Prozess der Karzinogenese mit Hilfe natürlicher oder synthetischer chemischer Substanzen. Chemoprotektiva besitzen die Fähigkeit, entweder die Schädigung der DNA zu verhindern oder die Proliferation bereits bestehender prämaligner Zellen zu unterbinden oder Reparaturprozesse zu fördern. Der Begriff der Chemoprävention ist noch relativ jung, er wurde im Jahre 1976 von Sporn in Anlehnung an den Begriff der Chemotherapie geprägt [1]. Die Chemoprävention hat in den letzten Jahren erheblich an Bedeutung gewonnen, da weltweit die Zahl der Krebserkrankungen kontinuierlich zunimmt, die Mortalitätsraten steigen, die therapeutischen Möglichkeiten jedoch im Vergleich dazu zurückbleiben. Die Chemoprävention ist daher für eine Vielzahl von soliden Tumoren ebenso wichtig wie eine Verbesserung der Früherkennungsmaßnahmen.

Tamoxifen war eine der ersten Substanzen, die als Chemoprotektivum in der Therapie des Mammakarzinoms Bedeutung erlangt hat. 1300 Frauen, bei denen ein erhöhtes Risiko bestand, an einem Mammakarzinom zu erkranken, erhielten über einen Zeitraum von 5 Jahren täglich randomisiert $20 \mathrm{mg}$ Tamoxifen bzw. Plazebo. Die Ergebnisse rechtfertigen den Einsatz dieser Substanz zur Chemoprävention, da das Risiko an einem Brustdrüsentumor zu erkranken, um 50\% gesenkt werden konnte [2]. Inzwischen stehen fast 2000 verschiedene Substanzen für den Einsatz in der Chemoprävention zur Verfügung und weitere Präparate werden folgen [3].

Es ist klar, dass Chemoprävention beim Bronchialkarzinom primär Aufgabe oder Reduktion des Nikotinabusus bedeutet. Vielen Rauchern ist aber eine Aufgabe des Nikotinabusus nicht möglich und auch Ex-Raucher sind über einen längeren Zeitraum einem erhöhten Tumorrisiko ausgesetzt, so dass bei diesen Patienten eine medikamentöse Chemoprävention indiziert wäre.

Immerhin sterben weltweit jährlich 1 Million Menschen an Lungenkrebs, und in der europäischen Union werden jedes Jahr 200000 neue Erkrankungsfälle und 145000 Todesfälle registriert [4]. Insbesondere in den Entwicklungsländern wird der Tabakkonsum weiter ansteigen, derzeit wird die Zahl der Raucher für das Jahr 2010 auf 1,3 Millionen Menschen geschätzt. Betrachtet man dabei die 5-Jahres-Überlebensrate, die z.Z. noch unter $10 \%$ liegt, dann wird deutlich, dass auch hier die Chemoprävention in den nächsten Jahren eine wichtige Rolle spielen sollte.

Im Folgenden soll aufgezeigt werden, welche Substanzen bislang in der Chemoprävention des Bronchialkarzinoms eingesetzt werden, welche Studien mit welchen Ergebnissen durchgeführt wurden und welche zukünftigen therapeutischen Möglichkeiten sich andeuten.

\section{Molekulargenetische Grundlagen}

Eine Voraussetzung für die Entwicklung von chemoprotektiven Substanzen ist eine detaillierte Kenntnis über den Mechanismus der Karzinogenese, wie sie in den letzten 20 Jahren gewonnen wurden [5].

Der Prozess der Karzinogenese erfordert 10-20 und mehr genetische Ereignisse, um eine normale Zelle in eine Krebszelle zu verwandeln [6]. Chronischer Nikotinabusus und andere Umweltnoxen führen über eine mutagene Schädigung des genetischen Materials zu einer Hemmung des programmierten Zelltodes, einer unkontrollierten Proliferation der Zelle, einer Neoangiogenese und zum Verlust der Zelldifferenzierung [7]. Die Zusammenhänge zwischen Nikotinabusus und Kanzerogenese sind in den letzten Jahren intensiv untersucht worden. Das Verständnis dieser Mechanismen führte zu einer neuen Strategie, um die Lungenkrebsinzidenz zu senken, besonders empfängliche Personengruppen zu definieren und neue Techniken in der Prävention zu entwickeln.

Im Zigarettenrauch sind über 200 Substanzen und mindestens 55 Karzinogene nachweisbar, insbesondere polyzyklische aromatische Kohlenwasserstoffe (PAH) und Nitrosamine (NNK), daneben andere Tumorpromotoren und Co-Karzinogene [8]. Metabolische Aktivierung von PAH und NNK führt u.a. zu einer Methylierung der DNA und zur Bildung von DNA-Komplexen (Addukten). Metabolische Aktivierung und Detoxifizierungsmechanismen halten sich dabei die Waage. Ihre individuell unterschiedliche Ausprägung bestimmt letztlich das individuelle Risiko der Krebsentstehung mit. Persistierende DNA-Addukte führen über Mutationen und andere Veränderungen des Genoms zur Karzinomentstehung. Hierbei besitzen Frauen nachweislich eine höhere Empfänglichkeit gegenüber den Karzinogenen im Tabakrauch als Männer $[9,10]$. Morphologisch führen die genetischen Veränderungen in mehreren Schritten von einer normalen Bronchialepithelzelle über hyperplastische und dysplastische Veränderungen zu einem Karzinoma in situ und schließlich zu einer manifesten Karzinomzelle (Multistep Carzinogenesis). Hierbei können bei gleicher Noxe multiple, genetisch unabhängige neoplastische Veränderungen des Epithels auftreten (Field Carzinogenesis) [7]. Bei Patienten mit Tumoren im Kopf/Halsbereich, insbesondere Larynxkarzinomen, besteht ein erhöhtes Risiko, zugleich an anderer Stelle in den Atemorganen bzw. dem oberen Gastrointestinaltrakt einen Zweittumor zu entwickeln [11].

Der Einsatz chemoprotektiver Substanzen kann in Kenntnis der molekulargenetischen Veränderungen in verschiedenen Phasen der Karzinomentstehung eingreifen, wobei beim Bronchialkarzinom zwischen primärer, sekundärer und tertiärer Prävention unterschieden werden kann. 
Primäre Chemoprävention bedeutet frühzeitige Intervention d.h. Aufgabe bzw. Reduktion des Nikotinabusus. Mittlerweile besteht kein Zweifel daran, dass Nikotinabusus als Hauptursache für die Entstehung von Lungenkrebs angesehen werden kann. Im Jahre 2000 sind in den USA 600 Billionen Zigaretten verkauft worden [12]. Das Risiko, an einem Lungenkrebs zu erkranken, reduziert sich in den ersten 5 Jahren nach Aufgabe des Rauchens nicht. Auch in den folgenden Jahren bleibt das relative Risiko, einen Lungenkrebs zu entwickeln, hoch und erreicht niemals dasjenige eines Nichtrauchers [13,14]. Durch Eliminierung inhalativer und anderer Noxen ist es allerdings möglich, das Krebsrisiko allgemein um $80-90 \%$ zu senken [15]. Genetische Prädispositionen sind nur in ca. $5 \%$ der Fälle für eine Krebsentstehung verantwortlich zu machen [16].

Sekundäre Prävention bedeutet bei Rauchern mit manifesten präkanzerösen Schleimhautveränderungen, wie intraepitheliale Neoplasien, Leukoplakien, Dysplasien usw. eine Reduktion des Risikos.

Das Ziel der tertiären Chemoprävention ist bei Patienten mit bereits resezierten Bronchialkarzinomen die Entwicklung eines

Tab. 1 Chemoprotektiva

Makro-Nutritiva

Nahrungsmittel- und Nahrungsmittelbestandteile

Mikro-Nutritiva

klassische Chemoprotektiva

moderne Chemoprotektiva

Retinoide und verwandte Substanzen natürliches Pro-Vitamin A

all-trans-Retinsäure (Tretinoin-ATRA)

13-cis-Retinsäure (13-cRA-Isotretinoin)

9-cis-Retinsäure (9-cR-Tretinoin)

Etretinat (synthetisches Pro-Vitamin A)

$\beta$-Carotine

Calcium

$\alpha$-Tocopherol (Vitamin E)

Vitamin C

Vitamin B12, Folsäure

Selen
Zweittumors zu verhindern. Definitionsgemäß entwickelt sich ein Zweittumor frühestens nach 3 Jahren. Er wird bei resezierten nichtkleinzelligen Bronchialkarzinomen in 2-4\% der Fälle beobachtet [17].

\section{Substanzen der Chemoprävention}

Die genetischen und morphologischen Veränderungen beim Bronchialkarzinom bieten Ansatzmöglichkeiten für eine Vielzahl von chemoprotektiven Substanzen, bei denen es sich um Nahrungsmittelbestandteile, spezielle chemische Verbindungen, Hormone, körpereigene Proteine, Antikörper, Wachstumsfaktoren u.a. handeln kann.

Tab. 1 gibt einen Überblick über die klassischen und die moderneren Chemoprotektiva, die bislang in Präventions-Studien eingesetzt wurden bzw. deren Wirksamkeit in Studien z.Z. untersucht wird. Ihre jeweiligen Angriffspunkte im Prozess der Karzinogenese sind in Abb. 1 dargestellt.

\section{Makro-Nutritiva: Obst und Gemüse}

Die vorliegenden epidemiologischen Untersuchungen und experimentellen Tierversuche geben Hinweise darauf, dass Chemoprävention zahlreicher Tumoren durch den Einsatz von Nahrungsmittelbestandteilen bzw. Nahrungsergänzungsstoffen bzw. extrahierten Substanzen aus Nahrungsmitteln möglich ist.

Zwischen 1983 und 2003 wurden insgesamt 37 Kohortenstudien und 59 Fall-Kontroll-Studien durchgeführt, um mögliche Zusammenhänge zwischen Obst- und/oder Gemüsekonsum bzw. der Zufuhr von Antioxidantien wie Vitamin C/A über die Nahrungsaufnahme einerseits und der Entwicklung von Lungenkrebs andererseits zu erfassen [18]. Der Beobachtungszeitraum dieser Studien lag zwischen 4 und 25 Jahren. Insgesamt wurden über 50000 Personen in den Studien untersucht. In fast allen Studien wurden Selbsterfassungsbögen eingesetzt, mit bis zu 23 Fragen zum Obst- und Gemüsekonsum. Die Mehrzahl der Studien belegen ein inverses Verhalten zwischen dem Auftreten von Lungenkrebs und dem Konsum von Obst und Gemüse sowohl bei Rauchern als auch bei Nicht- oder Exrauchern.

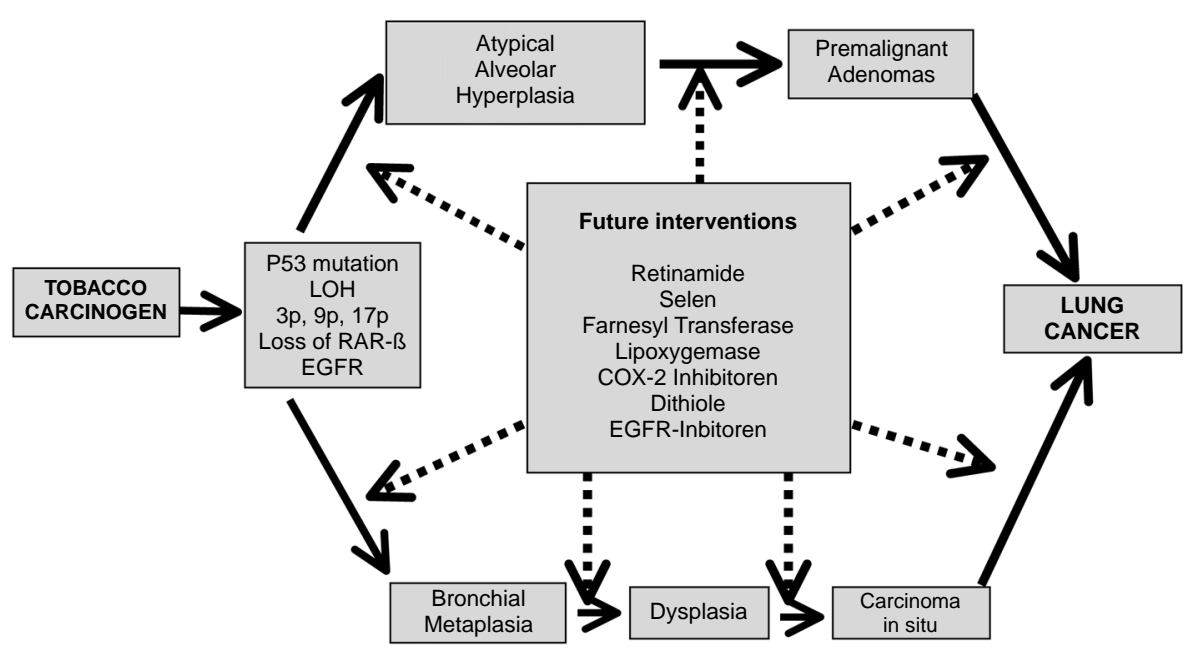

Abb.1 Zukünftige Strategien der modernen Chemoprotektiva, modifiziert nach Kim u. Mitarb. [27]. Dargestellt sind die verschiedenen Angriffspunkte der modernen Chemoprotektiva auf die frühen und möglicherweise noch reversiblen Stadien der Karzinogenese. 
Tab. 2 Molekulare Targets bzw. Biomarker der Chemoprävention $[27,35]$

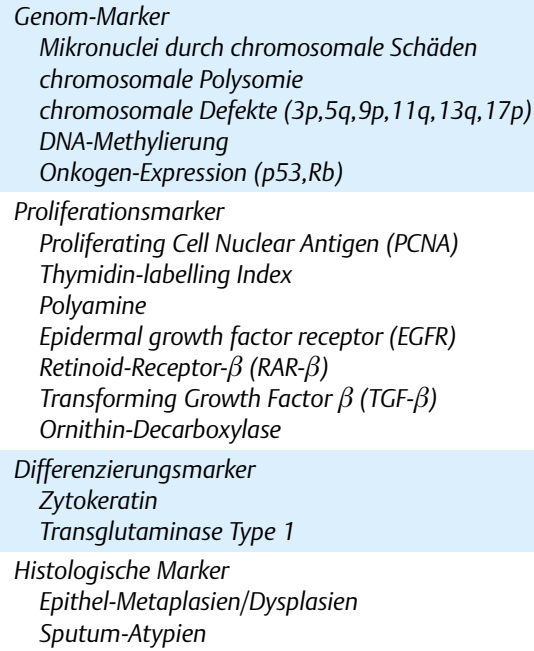

Trotz der Heterogenität der Studien mit einem Schwankungsbereich des 95\% Konfidenzintervalls zwischen 0,56 und 1,0, war das relative Risiko an einem Lungenkrebs zu erkranken unter obst- und/oder gemüsehaltigen Diäten im Mittel um 30\% abgesenkt. Unterschiede zwischen Männern und Frauen, den histologischen Subtypen oder den Rauchgewohnheiten bestanden nicht [19]. In Metaanalysen erbrachte der Obstkonsum geringfügig bessere Ergebnisse als der Gemüsekonsum [20].

\section{Mikro-Nutritiva}

\section{Retinoide und verwandte Substanzen}

Der Begriff der Retinoide umfasst das in pflanzlichen Substanzen vorkommende $\beta$-Carotin (Provitamin A) sowie die in tierischen Nahrungsmitteln vorhandenen Vitamin A-Vorstufen wie Retinol, Retinylester und Retinsäure.

Retinoide sind bislang die am besten untersuchten chemoprotektiven Substanzen. Vitamin A ist wichtig für Zellwachstum und -differenzierung und reguliert die Apoptose. Vitamin A-Mangel führt zur Störung der Zellhomöostase und bei Ratten zur Bildung von bronchoepithelialen Metaplasien, die denen chronischer Raucher ähneln. Epidemiologische Untersuchungen fanden ein inverses Verhalten zwischen dem Vitamin A-Spiegel und dem Auftreten bestimmter Neoplasien [21]. Über 1000 Retinoide sind bisher synthetisiert worden auf der Suche nach der wirksamsten Substanz mit der geringsten Toxizität [22].

$\beta$-Carotine werden in vivo zu Retinol umgewandelt. Da sie reichlich in der Nahrung vorhanden, zudem preiswert und nicht toxisch sind, werden sie bevorzugt in Studien eingesetzt, insbesondere auch wegen ihrer zusätzlichen antioxidativen Wirksamkeit.

Verantwortlich für die Wirkung der Retinoide auf den Zellstoffwechsel sind die 1987 aus dem Zellkern isolierten Retinoid-Rezeptoren (RXR und RAR-Rezeptoren mit den Untergruppen $\alpha, \beta$ und $\gamma$ ), die zur Superfamilie der Steroidrezeptoren gehören, zu der u.a. auch die Rezeptoren für Glukokortikoide, Thyroidhor- mone, Vitamin D, Progesteron und Östrogene gehören [23]. Das Verteilungsmuster der Retinoid-Rezeptoren variiert erheblich im normalen und neoplastischen Gewebe. In prämalignen Epithelveränderungen im Bereich der oberen Atemwege und in Bronchialkarzinomen lassen sich weniger RAR- $\beta$-Rezeptoren nachweisen als im normalen Gewebe [24].

Gehen Retinoid-Rezeptoren mit Retinoiden eine Bindung ein, so sind diese Komplexe in der Lage, über spezifische DNA-Sequenzen die Expression vieler Gene zu supprimieren oder zu aktivieren und somit positive, aber auch negative Wirkungen auf die Zelldifferenzierung, Zellteilung und Apoptose auszuüben. Einigen Retinoiden fehlt jedoch die Fähigkeit, mit Retinoid-Rezeptoren Bindungen einzugehen, bzw. sie werden möglicherweise zu bislang unbekannten Substanzen metabolisiert [25].

Hinweise für die antikanzerogenen Wirkung von Retinoiden ergaben sich bislang vor allem durch die erfolgreiche Therapie der Promyelozytenleukämie [26] und der reversiblen prämalignen Epithelveränderungen der oberen Atemwege von Rauchern. Parallel zur Gabe der Retinoide erfolgte eine Aufregulierung des RAR- $\beta$-Rezeptors [27].

Die Ergebnisse der bisherigen Studien zeigen Unterschiede zwischen Rauchern und Exrauchern in der Chemoprävention von Tumoren der Atemwege. Bemerkenswerterweise führten in der primären Prävention chronischer Raucher Tabakkonsum und die gleichzeitige Einnahme von Retinoiden (13-cRA und/oder $\beta$-Carotine) zu unerwünschten Interaktionen im Sinne einer $\mathrm{Zu}$ nahme der Lungenkrebsinzidenz und der Gesamtmortalität. Hierbei wurden in 3 großen Phase-III-Studien fast 7000 männliche Raucher über mehrere Jahre beobachtet. Die Probanden erhielten entweder Plazebo versus $\alpha$-Tocopherol und $\beta$-Carotin (ATBC: $\alpha$-Tocopherol, $\beta$-Carotene Study) [28] oder Plazebo versus $\beta$-Carotin und Retinylpalmitat (CARET: $\beta$ Carotene Retinol Efficacy Trial) [29] oder nur $\beta$-Carotin (Physician Health Study). Lungenkrebsinzidenz und Gesamtmortalität stiegen in der ATBC-Studie auf 18 bzw. 8\% und in der CARTET-Studie auf 28 und $17 \%$. Andererseits ergab die Physician Health Studie von 1996 bei Rauchern unter der Einnahme von $\beta$-Carotinen ein relatives Risiko von 0,93 an Lungenkrebs zu erkranken [30].

Die Nichtabnahme oder sogar Zunahme der Lungenkrebsinzidenz unter $\beta$-Carotinen bleibt ungeklärt. Viele Theorien werden diskutiert. Wahrscheinlich ist, dass $\beta$-Carotin bei Rauchern zu einer Methylierung des RAR- $\beta$-Promotors führen kann mit einer nachfolgenden Störung der Retinoid-Rezeptor-Gen-Interaktion [31].

In der sekundären Prävention führten Retinoide zu keiner Veränderung des Metaplasie-Index in der Bronchialschleimhaut; hierzu liegen 4 Phase-II-Studien vor [31]. Einzig eine Aufgabe des Nikotinabusus führt nachweislich zu rückläufigen Atypien im Sputum und in der Schleimhaut [31]. In der tertiären Prävention können Retinoide bei Exrauchern und Nichtrauchern jedoch einen regenerativen Effekt auf die Bronchialschleimhaut ausüben und zu einer Verringerung der lokoregionalen Rezidive führen; dies ist durch 3 Phase-III-Studien belegt [31]. 


\section{Selen}

Selen wird seit Jahren in der Prävention solider Tumoren, insbesondere dem Prostatakarzinom eingesetzt [32]. Selen besitzt antioxidative Eigenschaften. In Verbindung mit dem Enzym Glutathion-Peroxidase schützt Selen die Zelle vor Schäden an der DNA durch oxidativen Stress. Selen aktiviert den Reparaturmechanismus der DNA und kann dadurch die Etablierung von Mutationen verhindern [33]. Effekte auf das Immunsystem, das endokrine System, eine Hemmung der Proteinsynthese und eine Stimulierung der Apoptose werden ebenfalls postuliert.

Normalerweise wird ausreichend Selen über die Nahrung aufgenommen. Epidemiologische Studien konnten jedoch zeigen, dass ein inverses Verhältnis zwischen Selenkonzentration im Serum und der Entwicklung von Bronchialkarzinomen besteht [34]. Da anorganisches Selen und einige organische Selen-Verbindungen in hoher Konzentration (> $5 \mathrm{ppm}$ ) toxisch sein können, wird nach organischen Selenverbindungen gesucht, die in hohen Dosen zugeführt werden können, wie z.B. Methylenselenocyanat [27]. Beim Bronchialkarzinom lieferten die Studien mit Selensupplementierung bislang widersprüchliche Ergebnisse [27]. So läuft derzeit im Rahmen der ECOG eine Phase-III-Studie mit Selengabe zur sekundären Chemoprävention beim operierten NSCLC im Stadium I [31].

\section{$\alpha$-Tocopherol (Vitamin E)}

$\alpha$-Tocopherol ist Bestandteil aller Zellmembranen und wirkt über eine Hemmung der Lipidperoxidation als Antioxidanz. Epidemiologische Untersuchungen, Tierversuche und klinische Studien deuten auf eine krebspräventive Wirkung hin [35]. Prospektive Untersuchungen im Rahmen der ATBC-Studie konnten zeigen, dass bei jungen finnischen Rauchern, die eine erhöhte $\alpha$-Tocopherol-Konzentration im Serum aufwiesen, ein um 19\% geringeres Risiko bestand, an Lungenkrebs zu erkranken [36]. Es wird postuliert, dass $\alpha$-Tocopherol möglicherweise einige der Toxizitäten von Retinoiden (insbesondere 13 cRA) kompensieren kann [35].

\section{Zuküinftige Strategien}

Mit Fortschritten auf dem Gebiet der molekularen Genetik von Krebserkrankungen haben sich viele neue therapeutische Möglichkeiten ergeben, von denen die wichtigsten hier aufgeführt werden sollen. Ergebnisse klinischer Studien liegen bislang noch nicht oder nur in vorläufiger Form vor.

\section{Cyclooxygenase-Inhibitoren}

In vielen menschlichen Tumoren lassen sich erhöhte Konzentrationen von Prostaglandinen nachweisen [37]. Das Enzym Cyclooxygenase (COX), mit den Isoenzymen COX-1 und COX-2, katalysiert den Oxygenisierungsprozess der Arachidonsäure. Cyclooxygenase-2 ist in normalem Gewebe nicht nachweisbar und bedarf entzündlicher Stimuli wie Zytokinen, Wachstumsfaktoren u.a. [38]. COX-2 lässt sich vermehrt in prämalignen Geweben und malignen Tumoren nachweisen. Verschiedene Pathomechanismen der Karzinogenese werden diskutiert: Immunsuppression über die Interleukine 10 und 12, Hemmung der Apoptose, chronische Entzündung, gesteigerte Angiogenese, sowie eine Rolle von COX-2 im Prozess der Proliferation, Invasion und Me- tastasierung $[39,40]$. Erhöhte COX-2-Aktivitäten lassen sich in prämalignen Veränderungen der Lunge, bronchiolo-alveolären Karzinomen und Adenokarzinomen nachweisen [41]. Erhöhte COX-2-Konzentrationen könnten auf die Entwicklung eines Adenokarzinoms hindeuten. Nikotin induziert die Expression von COX-2, und Nitrosamine (NNK) werden teilweise durch COX-2 zu bioaktiven Substanzen metabolisiert. COX-2-Inhibitoren hemmen im Tierversuch die Karzinogenese, z. B. wird Celecoxib, ein selektiver COX-2-Inhibitor, derzeit im Rahmen einer Studie bei Rauchern in der sekundären Prävention getestet. [42].

\section{Lipoxygenase-Inhibitoren}

In Zellkulturen, allerdings noch nicht in klinischen Studien, konnte gezeigt werden, dass Produkte der 5-Lipoxygenase wie Leukotriene und Hydroxyeicosatetrasäuren Einfluss haben auf das Wachstum von Lungenkrebszellen [27]. 5-Lipoxygenase wird stimuliert durch Gastrin-Releasing Peptide (GRP) und Insulin-like Growth Faktors (IGF). Entsprechend können Lipoxygenase-Inhibitoren die Zellproliferation hemmen [43 - 45]. Inhibitoren wie Zafirlukast, das für das Asthma entwickelt wurde, haben sich in präklinischen Studien auch als chemopräventiv beim Lungenkrebs erwiesen [46]. Weitere Studien sind jedoch erforderlich, um diese Beobachtung zu validieren.

\section{Epidermaler Wachstumsfaktorrezeptor}

Epidermale Wachstumsfaktorrezeptoren (EGFR/erbB1), die zu den Tyrosinkinasen gehören, lassen sich in prämalignen Bronchialschleimhautveränderungen nachweisen und stimulieren das Wachstum normaler und maligner Zellen [47]. Von der normalen Bronchialschleimhaut über die Hyperplasie und Dysplasie bis hin zum Karzinom nimmt die Expression von EGFR/erbB1 zu, so dass angenommen werden kann, dass erbB1 in der Karzinogenese eine bedeutende Rolle spielt [48]. HER2/erbB2 wird hingegen weniger stark exprimiert. EGFR wird vermehrt von nichtkleinzelligen Tumorzelllinien exprimiert [49]. In klinischen Versuchen ergab die Behandlung mit cis-Retinoiden in der Prävention von Bronchialkarzinomen jedoch keine Veränderungen der EGFR-Expression [50]. Präklinische Untersuchungen zeigten, dass durch Tyrosinkinasehemmer das Tumorwachstum gehemmt werden kann [51]. Am National Cancer Institut, New York, wird derzeit (Gefitinib) versus Plazebo geprüft. Endpunkte sind die Reversibilität der morphologischen Veränderungen und die Expression bestimmter Marker wie Ki67, EGFR/erbB1, HER 2 u. a. [31]. Weiterführende klinische Studien stehen noch aus.

\section{Farnesyltransferase-Inhibitoren}

Farnesyltransferase steuert die biologische Funktion der ras-Proteine, entsprechend blockieren Farnesyltransferase-Inhibitoren die Funktion der ras-Proteine und anderer Proteine. In Zellkulturen und Tierversuchen zeigten Farnesyltransferase-Inhibitoren eine Antitumoraktivität [52]. Ergebnisse einer klinischen Studie mit einem Farnesyltransferase-Hemmer (R11777) stehen noch aus [53].

\section{Dithiole}

Oltipraz(-4-methyl-5-pyrazinyl-1,2-dithiol-3-thion) und Anethiol-dithiolethion (ADT; 5-[(4-methoxyphenyl-1-propenyl] -1,2-dithiol-3-thion) können die intrazellulären Konzentrationen der Glutathion-S-Transferase (GST) erhöhen und damit als Antioxidantien wirken. ADT wurde in einer randomisierten Pha- 
se-II-Studie bei Rauchern mit einer bronchialen Dysplasie eingesetzt [54]. Histologische Veränderungen der Bronchialschleimhaut, kontrolliert mit der Autofluoreszenz-Bronchoskopie, zeigten signifikant weniger neue dysplastische Läsionen und eine geringere Progression der bereits existierenden dysplastischen Veränderungen.

\section{Retinamide (synthetische Retinoide, Fenretinide)}

Nachdem die bisherigen Therapien mit Retinoiden bei Rauchern keinen Einfluss auf die prämalignen Veränderungen der Bronchialschleimhaut gezeigt haben, möglicherweise durch eine Inaktivierung der Retinoidrezeptoren (s. o.), wurden synthetische Retinoide, so genannte Retinamide, entwickelt. Diese weisen einen Wirkmechanismus auf, der teilweise unabhängig von den Retinoidrezeptoren arbeitet [55]. In einer Phase-II-Studie bei Rauchern besaß Fenretinide allerdings keinen Einfluss auf den dysplastischen Bronchialschleimhaut-Index [56].

\section{Biomarker}

Biomarker sind definitionsgemäß Substanzen, die sich in Körperflüssigkeiten, Zellen oder Geweben nachweisen lassen, und mit deren Hilfe frühzeitig umweltbedingte Schäden erkannt werden können. Biomarker können sowohl selbst „molecular targets“ sein als auch „intermediate markers“, die den Prozess der Krebsentstehung anzeigen, ohne selbst direkt beteiligt zu sein. Mit Hilfe von Biomarkern lassen sich heute Studien zur Krebsprävention weniger zeitaufwändig und kostenintensiv als bisher an einem relativ kleinen Patientenkollektiv durchführen und dabei die Aussagen zur Krebsinzidenz und zum Überleben biochemisch unterstützen. Biomarker beim Bronchialkarzinom betreffen sowohl morphologische bzw. histologische als auch genetische und molekulare Veränderungen (Tab. 2). Zahlreiche Studien haben sich mit dem Metaplasie-Index der Bronchialschleimhaut bei Rauchern beschäftigt [57,58]. Der Metaplasie-Index ist abhängig vom Rauchverhalten (pack-years), von der Schwere der chronisch-obstruktiven Bronchitis und den Sputum-Atypien. Mit Hilfe der Autofluoreszenzbronchoskopie kann die Aussagekraft noch verbessert werden. Welche prognostische Bedeutung die verschiedenen präneoplastischen Veränderungen der Bronchialschleimhaut haben, ist aufgrund der geringen Datenlage z.Z. noch nicht geklärt.

Genetische Marker, wie p53, EGFR und HER 2 lassen sich zwar immunhistochemisch nachweisen, ihre Validität als Biomarker ist jedoch durch klinisch-pathologische Studien noch nicht belegt. Neueste Entwicklungen beschäftigen sich mit der Genexpression und somatischer Mutationen sowie der Proteom-Analyse [31]. Es konnte bereits gezeigt werden, dass komplexe Proteinprofile ein vielversprechendes Potenzial für die Früherkennung des Prostatakarzinoms bergen [59].

\section{Diskussion}

Eine erfolgreiche medikamentöse Chemoprävention gibt es bislang nur für wenige Tumoren [60]. Die Ergebnisse von Chemopräventions-Studien beim Bronchialkarzinom sind bislang eher enttäuschend. Große Studien mit Retinoiden zeigten sogar eine
Zunahme der Lungenkrebsinzidenz, obgleich große epidemiologische Untersuchungen ergaben, dass Vitamine die Lungenkrebsrate senken können. Hierbei ist an die vielfältigen Interaktionsmöglichkeiten innerhalb des Genoms zu denken, bei dem eine Substanz bzw. ein genetischer Polymorphismus bei gleichzeitiger Aktivierung verschiedener Gene einmal die Aktivierung eines Gens und ein anderes Mal eine Suppression bewirken können. Die relevanten Fakten sind somit vermutlich im Feld der Epigenetik und der Epistasis zu suchen.

Dennoch haben die Studien zu Retinoiden wichtige Erkenntnisse erbracht, vor allem dass Unterschiede zwischen Rauchern und Ex-Rauchern in der Empfänglichkeit gegenüber Chemoprotektiva bestehen. In den USA (für die Zahlen vorliegen) gibt es beispielsweise 45 Millionen Ex-Raucher, die ein erhöhtes Risiko besitzen, in den nächsten 10 Jahren ein Bronchialkarzinom zu entwickeln [61]. Diese Patienten könnten erfolgversprechend mit chemoprotektiven Substanzen behandelt werden. Studienansätze (National Cancer Institute, NY) sind vorhanden [7,31,62]. Derzeit sind jedoch eine Vielzahl von Fragen zur konkreten Durchführung noch offen.

Die erste dieser Fragen ist, welche Substanzen primär eingesetzt werden sollten und in großen Mengen eingesetzt werden können, ferner in welcher Kombination, falls eine solche einen Vorteil mit sich bringt. Die Molekulargenetik hat eine Vielzahl von Substanzen vorgeschlagen oder bereits entwickelt, die theoretisch zur Prävention eingesetzt werden können und die sich derzeit in klinischen Prüfungen an fortgeschrittenen Bronchialkarzinomen befinden.

Eine weitere Frage ist, welche Biomarker erfolgreich in der Analyse prämaligner Veränderungen der Bronchialschleimhaut eingesetzt werden können. Anders als die histologischen Veränderungen, die mit der Aufgabe des Nikotinabusus rückläufig sind, persistieren die molekular-genetischen Veränderungen über einen längeren Zeitraum. Hier bieten sich Möglichkeiten mit Hilfe von Genchips und Proteomanalysen sensitive Marker oder Muster von Markern zu finden.

Eine dritte und wesentliche Frage ist, welche Risikogruppen für eine Intervention an erster Stelle gewählt werden sollten. Zum einen könnten das Ex-Raucher sein. 50\% aller Bronchialkarzinome entwickeln sich bei Ex-Rauchern [61]. Die bisherigen Studien belegen, dass Chemoprävention bei Ex-Rauchern effektiv ist. Zum anderen besitzen Frauen eine größere Empfänglichkeit gegenüber kanzerogenen Substanzen im Tabakrauch. Ferner können Risikopatienten als solche Patienten definiert werden, die 20-30 Jahre geraucht haben, die Diagnose einer COPD besitzen und bei denen sich atypische Veränderungen im Sputum nachweisen lassen. Auch operierte Patienten mit einem nichtkleinzelligen Bronchialkarzinom in frühen Tumorstadien können zu den Risikopatienten gezählt werden, bei denen eine Chemoprävention zu erwägen ist [63].

Die vierte Frage gilt der Applikationsform. Optimal wäre eine inhalative Therapie, die eine hohe lokale Konzentration in der Lunge sichert [64]. Budesonid in Aerosolform war die erste inhalative Substanz, die in der Prävention des Bronchialkarzinoms er- 
probt wurde [65]. Ferner scheinen liposomale Retinoide (ATRA) in ersten Versuchen Erfolg versprechend [66].

Zusammenfassend lässt sich feststellen, dass in den letzten Jahren große Fortschritte auf dem Gebiet der Krebsprävention erzielt wurden; dies gilt sowohl für die einzusetzenden Substanzen als auch für relevante Biomarker und ein optimales Studiendesign. Es wird in Zukunft vermutlich möglich sein, klinische Studien von Risikopatienten in kürzeren Zeiträumen und mit kleineren Fallzahlen als bislang durchzuführen. Damit kann allen Beteiligten, insbesondere den Patienten, aber auch der forschenden Industrie ein überschaubarer Zeitraum angeboten werden, in dem Ergebnisse erzielt werden. Es ist somit zu hoffen, dass in absehbarer Zeit klinische Studien weitere Fortschritte auf dem Gebiet der Chemoprävention erbringen werden.

\section{Literatur}

${ }^{1}$ Sporn MB, Dunlop NM, Newton DL et al. Prevention of chemical carcinogenesis by vitamin A and its synthetic analogs (retinoids). Fed Proc 1976; 35: $1332-1338$

2 Fisher B, Costantino JP, Wickerham Dl et al. Tamoxifen for prevention of breast cancer. J Natl Cancer Inst 1998; 90: 1371-1388

${ }^{3}$ Lippman SM, Benner SFE, Hong WK. Cancer chemoprevention. J Clin Oncol 1994; 12: $851-873$

${ }^{4}$ Sant M, Aareleid T, Berrino F et al. EUROCART-3: survival of cancer patients diagnosed 1990-1994 results and commentary Oncol 2003; 14 (suppl 5): $61-118$

${ }^{5}$ Perera FP. Molecular epidemiology: Insight into cancer susceptibility, risk assessment and prevention. J Natl Cancer Inst 1996; 88: 496-509

${ }^{6}$ Bartsch H, Petruzelli S, De Flora S et al. Carcinogen metabolism in human lung tissues and the effect of tobacco smoking: results from a case-control multicenter study on lung cancer patients. Environ Health Perspect 1992; 98: 119-124

${ }^{7}$ Soria J-C, Kim ES, Fayette J et al. Chemoprevention of lung cancer. The Lancet Oncol 2003; 4: 659-669

${ }^{8}$ Hecht SS. Tobacco Smoke Carcinogens and Lung Cancer. J Natl Cancer Inst 1999; 91: 1194-1210

${ }^{9}$ Henschke CI, Miettinen OS. Womens susceptibility to tobacco carcinogens. Lung Cancer 2004; 43: 1 -5

10 Zang EA, Wynder EL. Differences in lung cancer risk between men and women: examinations of the evidence. J Natl Cancer Inst 1996; 88: $183-192$

${ }^{11}$ Christensen P, Joergensen K, Munk J. Hyperfrequency of pulmonary cancer in a population of 415 patients treated with laryngeal cancer. Laryngoscope 1987; 97: 612-614

12 Mackay J, Erickson M. The Tobacco Atlas. WHO 2002: 1-128

13 Shottenfeld D, Fraumeni Jr JF. Cancer epidemiology and prevention, 2nd edn. New York: Oxford University Press, 1996

${ }^{14}$ McLaughin JK, Hubrec Z. Smoking and cancer mortality among US veterans: a 26-year follow-up. Int J Cancer 1995; 6: 190-193

${ }^{15}$ Lippman SM, Waun KH, Benner SE. The chemoprevention of cancer In: Greewald P, Kramer BS, Weed DL (eds.). Cancer Prevention and Control. New York: Marcel Dekker, 1995: 329-352

${ }^{16}$ Tyczynski JE, Bray F, Parkin DM. Lung cancer in Europe in 2000: epidemiology, prevention, and early detection. The Lancet Oncology 2003; 4: $45-55$

${ }^{17}$ Pastorino U, Infante M, Maioli M et al. Adjuvant treatment of stage I lung cancer with high-dose vitamin A. J Clin Oncol 1993; 11: $1216-1222$

${ }^{18}$ IARC. Handbooks of Cancer Prevention. Vol. 8. World Health Organization, 2003: $81-84$

${ }^{19}$ Smith-Warner SA, Spiegelman D, Yuan S-S et al. Fruits, vegetables and lung cancer: a pooled analysis of cohort studies. Int J Cancer 2003; 107: $1001-1011$

${ }^{20}$ Riboli E, Norat T. Epidemiological evidence of the protective effect of fruit and vegetables on cancer risk. Am J Clin Nutr. 2003; 78 (Suppl): 559S-569S
${ }^{21}$ Hong WK, Lippmann SM, Itra LM. Retinoids and human cancers. In: Sporn MB, Roberts AB, Goodman DS (eds). The Retinoids. New York: Raven Press, 1994: 597-624

${ }^{22}$ Lippman SM, Heyman RA, Kurie JM et al. Retinoids and chemoprevention: clinical and basic studies. J Cell Biochem 1995; 22: 1-27

${ }^{23}$ Mangelsdorf D, Umesono K, Evans RM. The retinoid receptor. In: Spron MB, Roberts AB, Godman DS (eds.). The Retinoids:Biology, Chemistry, and Medicine. New York, NY: Raven Press Ltd., 1994: 319-349

${ }^{24} \mathrm{Xu} \mathrm{X}-\mathrm{C}$, Sozzi G, Lee JS et al. Suppression of retinoic acid receptor $\beta$ in non-small-cell lung cancer in vivo implications for lung cancer development. J Natl Cancer Inst 1997; 89: 624-629

${ }^{25}$ Smith MYA, Parkinson DR, Cheson BD et al. Retinoids in cancer therapy. J Clin Oncol 1992; 10: 839-864

${ }^{26} \mathrm{Hu}$ L, Crowe DL, Rheinwald JG. Abnormal expression of retinoic acid receptors and keratin 19 by human oral and epidermal squamous cell carcinoma cell lines. Cancer Res 1991; 51: 3972-3981

${ }^{27}$ Kim ES, Hong WK, Khuri FR. Prevention of lung cancer. Chest Surg Clin North Am 2000; 10: 663-690

28 The $\alpha$-Tocopherol, $\beta$-Carotene Cancer Prevention Study Group. The effect of vitamin $E$ and $\beta$-carotene on the incidence of lung cancer and other cancers in male smokers. N Engl J Med 1994; 330: 1029- 1035

29 Omenn GS, Goodman GE, Thornquist MD et al. Effects of a combination of $\beta$-carotene and vitamin A on lung cancer and cardiovascular disease. N Engl J Med 1996; 334: 1150-1155

${ }^{30}$ Hennekens $\mathrm{CH}$, Buring JE, Manson JE et al. Lack of long-term supplementation with $\beta$-carotene on the incidence of malignant neoplasms and cardiovascular disease. N Engl J Med 1996; 334: 1145-1149

31 Winterhalder RC, Hirsch FR, Kotantoulos GK et al. Chemoprevention of lung cancer - from biology to clinical reality. Ann Oncol 2004; 15: $185-196$

32 Clark LC, Dalkin B, Krongard A et al. Decreased incidence of prostate cancer with selenium supplemetation: results of a double-blind cancer prevention trial. Br J Urol 1998; 81: 730-734

${ }^{33}$ Van den Brandt PA, Goldbohm RA, vant Veer P et al. A prospective cohort study on selenium states and the risk of lung cancer. Cancer Res 1993; 53: $860-4865$

${ }^{34}$ Reid ME, Duffield-Lillico AJ, Garland L et al. Selenium supplementation and lung cancer incidence: an update of the nutritional prevention of cancer trial. Cancer Epidemiol Biomark Prev 2002; 11: $1285-1291$

35 Show GL. Carcinogenesis and lung cancer. In: Kane MA, Bunn Jr. PA. Biology of Lung Cancer. Marcel Dekker, 1998: 99-117

${ }^{36}$ Woodson K, Tangrea JA, Barrett MJ et al. Serum $\alpha$-Tocopherol and subsequent risk of lung cancer among male smokers. J Natl Cancer Inst 1999; 91: $1738-1743$

${ }^{37}$ Dannenberg AJ, Altorki NK, Subbaramarah K. Selective inhibitors of COX-2: new applications in oncology. Educational Book ASCO 2001: $21-27$

${ }^{38}$ Herschman HR. Prostaglandin synthase 2. Biochim Biophys Acta 1996; 1299: $125-140$

39 Kitayama W, Denda A, Yoshida J. Increased expression of cyclooxygenase-2 protein in rat lung tumors induced by N-nitrosobis (2-hydroxypropyl) amine. Cancer Lett 2000; 148: 145-152

${ }^{40}$ EL Bayoumy K, Iatropoulos M, Amin S et al. Increased expression of cyclooxygenase 2 in rat lung tumors induced by the tobacco specific nitrosamine 4-(methylnitrosamino)-4-(3-pyridyl)-1-butanone: the impact of a high fat diet. Cancer Res 1999; 59: 1400-1403

${ }^{41}$ Ramalingam S, Belami CP. Cyclooxygenase- 2 inhibitors in lung cancer. Clin Lung Cancer 2004; 5: 245-253

42 Hosomi Y, Yokose T, Hirose Y et al. Increased cyclooxygenase 2 (COX-2) expression occurs frequently in precusor lesions of human adenocarcinoma of the lung. Lung Cancer 2000; 30: 73-81

${ }^{43}$ Avis IM, Jett M, Boyle T et al. Growth control of lung cancer by interruption of 5-lipoxygenase mediated growth factor signaling. J Clin Invest 1996; 97: 806-813

${ }^{44}$ Moody TR, Layton J, Martinez A et al. Lipoxygenase inhibitors prevents lung carcinogenesis and inhibit non-small cell lung cancer growth: Exp. Lung Res 1998; 24: 617-628

${ }^{45}$ Rioux N, Castonguay A. Inhibitors of lipoxygenase: a new class of cancer chemoprevention agents. Carcinogenesis 1998; 19: 1393-1400

${ }^{46}$ Soriano AF, Helfrich B, Chan DC et al. Synergistic effects of new chemopreventive agents and conventional cytotoxic agents against human lung cancer cell lines. Cancer Res 1999; 59: 6178-6184 
${ }^{47}$ Kurie JM, Shin HJ, Lee JS et al. Increased epidermal growth factor receptor expression in metaplastic bronchial epithelium. Clin Cancer Res 1996; 2: 1787-1793

${ }^{48}$ Piythilake CJ, Frost AR, Manne U et al. Differential expression of growth factors in squamous cell carcinoma and precancerous lesions of the lung. Clin Cancer Res 2002; 8: 734-744

${ }^{49}$ Franklin WA, Veve R, Hirsch FR et al. Epidermal growth factor receptor family in lung cancer and premalignancy. Sem Oncol 2002; 29 (suppl 4): $3-14$

${ }^{50}$ Xia W, Mullin RJ, Keith BR et al. A dual tyrosine kinase inhibitor blocks EGF activation of EGFR/erbB2 and downstream Erk 1/2 and AKT pathways. Oncogene 2002; 21: 6255-6263

${ }^{51}$ Hirsch FR, Scagliotti GV, Langer CJ et al. Epidermal growth factor family of receptors in preneoplasia and lung cancer: perspectives for targeted therapies. Lung Cancer 2003; 41: 452 -542

52 Sebti SM, Ajei AA. Farnesyltransferase inhibitors. Sem Oncol 2004; 31 (Suppl 1): 28-39

${ }^{53}$ Wright JJ, Zerivitz K, Gravell AE et al. Clinicals trials referral source. Current clinical trials of R115777 (Zarnestra). Oncology (Huntingt) 2002; 16: 930-937

${ }^{54}$ Lam S, MacAuley C, le Riche JC et al. A randomized phase IIb trial of Anethol dithiolethione in smokers with bronchial dysplasia. J Natl Cancer Inst 2002; 94: 1001 - 1009

${ }^{55}$ Dimitrovsky E. N-(-4-hydroxyphenyl) retinamide activation of a distinct pathway signaling apoptosis. J Natl Cancer Inst 1997; 89: $1179-1181$

${ }^{56}$ Kurie JM, Lee JS, Khuri FR et al. N-(-4-hydroxyphenyl) retinamide in the chemoprevention of squamous metaplasia and dysplasia of the bronchial epithelium. Clin Cancer Res 2000; 6: 2973-2979
${ }^{57}$ Kurie JM, Lee JS, Morice RC et al. Autofluorescence bronchoscopy in the detection of squamous metaplasia and dysplasia in current and former smokers. J Natl Cancer Inst 1998; 90: 991 - 995

${ }^{58}$ Hirsch FR, Prindiville SA, Miller YE et al. Fluorescence versus whitelight bronchoscopy for detection of preneoplastic lesions: a randomized study. J Natl Cancer Inst 2001; 93: 1385-1391

${ }^{59}$ Banez LL. Diagnostic potential of serum proteomics patterns in prostate cancer. J Urol 2003; 170: $442-446$

${ }^{60}$ Kim ES, Khuri FR, Hong WK. Chemoprevention trials. In: Bertino JR (eds.). Encyclopedia of Cancer. Vol 1. 2 ed. Academic Press, 2002: 457-472

${ }^{61}$ Burns DM. Primary prevention, smoking and smoking cessation for future trends in lung cancer. Cancer 2000; 89: 2506-2509

${ }^{62}$ Mushine JL, Hirsch FR. Lung cancer chemoprevention: moving from concept to a reality. Lung Cancer 2003; 41: S163 - S174

${ }^{63}$ Martini N, Bains MS, Burt ME et al. Incidence of local recurrence and second in resected stage I lung cancer. J Thorac Cardiovasc Surg 1995; 301: $120-129$

${ }^{64}$ Sharma S, White D, Imondi AR et al. Development of inhalational agents for oncological use. J Clin Oncol 2001; 9: 1839-1847

${ }^{65}$ Wattenberg LW, Wiedmann TS, Estensen RD et al. Chemoprevention of pulmonary carcinogenesis by aerosolized budesonide in female $\mathrm{A} / \mathrm{J}$ mice. Cancer Res 1997; 57: 5489-5492

66 Parthasarathy RR, Gilbert B, Mehta K. Aerosol delivery of liposomal alltrans-retinol acid to the lungs. Cancer Chemother Pharmacol 1999; 43: $277-283$ 